\title{
Coronal Magnetic Field Topology over CME Productive Quiescent Filaments
}

\author{
Yan $\mathrm{Li}^{1}$ and Janet Luhmann ${ }^{1}$ \\ ${ }^{1}$ Space Sciences Laboratory, University of California, 7 Gauss Way, Berkeley, CA 94720, USA
}

\begin{abstract}
Magnetic field topology at the CME source regions is considered to play a role in CME initiation processes. We use observational data and Potential Field Source Surface model to investigate the magnetic field topology over CME productive quiescent filaments. We found both bipolar topology and quadrupolar topology at CME source regions, but bipolar topology is more common overall and in each year. The total occurrence ratio between bipolar and quadrupolar topology is about 3:1 with our 80 events. On the rising phase of the solar cycle, there is mostly bipolar topology, but on the declining phase, there is a mixture of both bipolar and quadrupolar topology.
\end{abstract}

Keywords. Sun: magnetic fields, Sun: coronal mass ejections (CMEs), Sun: corona, Sun: filaments

CME initiations are still a mystery and debates between CME models are inconclusive (Antiochos 1998, Antiochos et al. 1999, Forbes, 2000, Linker et al. 2001, 2003). The so-called Breakout model (Antiochos, 1998; Antiochos et al., 1999) requires a quarupolar topology of the magnetic field at a CME initiation site. Instead of opening the overlying field lines to let out an eruption, the "breakout" process transfers the overlying closed field lines to the side arcades through magnetic reconnection. In order for the reconnection to take place, some anti-parallel components between the overlying field arcade and the lower central field arcade are needed. On the other hand, Linker et al. (2001, 2003) showed with MHD simulations that adequate amount of flux cancellation at the magnetic neutral line at the base of a bipolar region may also lead to the eruption of a sheared structure and the overlying arcade. All closed field arcades over the sheared structure are set in one direction, or parallel in their model. They pointed out that the flux cancellation decreases the open field energy of the system. We also name the two topologies anti-parallel and parallel topology, respectively, referring to the relationship between the erupting small arcade and the overlying large scale arcade. Using observational data and PFSS model we test what kind of coronal magnetic topology is most responsible for spawning CMEs. CMEs are commonly found associated with filament disappearances (Webb 2000, Cremades and Bothmer 2004, Jing et al., 2004).

We analyzed a group of 80 quiescent filament related CMEs over 1996 to 2004. These CMEs are wide CMEs with angular widths $\geqslant 80^{\circ}$ and the filaments at the CME source regions are located under large scale closed arcades. Our data source includes the LASCO CME catalog (Yashiro et al. 2004) at http://cdaw.gsfc.nasa.gov/CME_list/ by Gopalswamy and Yashiro, BBSO $\mathrm{H}_{\alpha}$ images at http://www.bbso.njit.edu/, MWO synoptic maps and PFSS coefficients (R. Ulrich and C.N. Arge, personnal communication), and $\mathrm{MDI}$ and $\mathrm{GONG}^{+}$magnetograms.

Two examples are given below to illustrate the magnetic field topologies found through our analyses. Fig. 1 shows the magnetic field topology at the source region of a filament related CME on 1997/10/23 at 11:26:50 UT (see the CME catalog details), included are projections of field lines around the filament longitudes and a partial MDI magnetogram. 

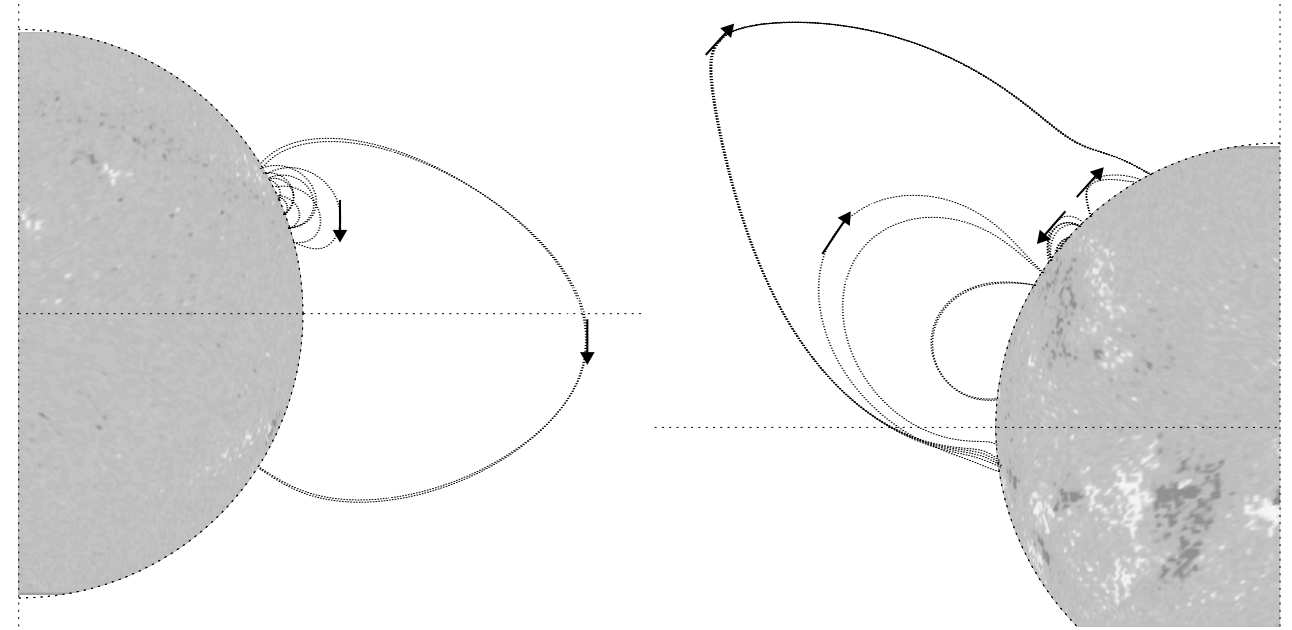

Figure 1. Projections of field lines at the filament longitudes are shown with MDI magnetograms. The arrows indicate the field directions. Magnetic field topologies at the source regions of filament related CMEs on 1997/10/23 at 11:26:50 UT (left) and on 2003/01/20 at 18:30:05 UT (right) show a bipolar (left) and a qadrupolar (right) topology, respectively.

The arrows indicate the field directions of the field line arcades, and in this case the lower arcades and the upper arcades have parallel fields, a bipolar source region. In Fig. 2 the same analyses for a CME on 2003/01/20 at 18:30:05 UT. But now a quadrupolar source region is evident, where between two side arcades the central small arcades (over the filament channel) have a field direction that is anti-parallel to that of the overlying upper arcades.

Among our $80 \mathrm{CME}$ events, we found that bipolar topology is more common overall and in each year. The total occurrence ratio between bipolar and quadrupolar topology is about $3: 1$. On the rising phase of the solar cycle, there is mostly bipolar topology, but on the declining phase, there is a mixture of both bipolar and quadrupolar topology. The bipolar topology occurrence has no clear solar cycle dependence. The quadrupolar topology occurrence peaks on the declining phase in the current solar cycle 23.

\section{Acknowledgements}

Supported by the NSF/CISM fund, DoD/MURI fund and NSF/ATM-0451438 award.

\section{References}

Antiochos, S. K., 1998, ApJ 502, L181.

Antiochos, S. K., DeVore, C. R., \& Klimchuk, J. A. 1999, ApJ 510, 485-493.

Cremades, H., \& Bothmer, V., 2004, AA 422, 307-322.

Forbes, T. 2000, JGR 105, 23153-23166.

Jing, J., Yurchyshyn, V.B., Yang, G., Xu, Y., \& Wang, H., 2004, ApJ 614, 1054-1062.

Linker, J. A., Lionello, R., Mikic, Z., \& Amari, T., 2001, JGR 106, 25165-25176.

Linker, J A., Mikic, Z., Lionello, R., Riley, P., Amari, T., \& Odstrcil, D. 2003, PhPl 10, 1971.

Webb, D. F. 2000, JATP, 62, 1415.

Yashiro, S., Gopalswamy, N., Michalek, G., St. Cyr, O. C., Plunkett, S. P., Rich, N. B., \& Howard, R. A., 2004, JGR 109, CiteID A07105. 